\title{
Fractional Delayer Utilizing Hermite Interpolation with Carathéodory Representation
}

\author{
Qiang DU, Yaoliang SONG, Zeeshan AHMAD \\ School of Electronic and Optical Engineering, Nanjing University of Science and Technology, \\ Xiaolingwei Street 200, 210094, Nanjing, China \\ qdu1023@gmail.com,ylsong@njust.edu.cn,dr.zeeshan@njust.edu.cn \\ Submitted September 13, 2017 / Accepted December 12, 2017
}

\begin{abstract}
Fractional delay is indispensable for many sorts of signal processing applications and fractional delay filter (FDF) is a straightforward method to delay discrete signals. FDF based on Hermite interpolation with an analog differentiator has a low time-domain error, but a more complicated sampling module than Shannon sampling scheme. A simplified scheme, which is based on Shannon sampling and utilizing Hermite interpolation with a digital differentiator, will lead to a much higher time-domain error when the signal frequency approaches the Nyquist rate. In this letter, we propose a novel fractional delayer utilizing Hermite interpolation and Carathéodory representation. The samples of differential signal are obtained by Carathéodory representation from the samples of the original signal only. So only one sampler is required and the sampling module is simple. Simulation results for four types of signals demonstrate that the proposed method has significantly higher interpolation accuracy than Hermite interpolation with digital differentiator.
\end{abstract}

\section{Keywords}

Delayer, fractional delay, Hermite interpolation, Carathéodory representation

\section{Introduction}

Fractional delayer could delay a discrete signal by a fraction of the sampling period and has taken a key role in many applications such as communications, array signal processing, sampling-rate conversion, speech coding and synthesis, etc. [1-5]. Fractional delay filter (FDF) is an intuitive and straightforward system to delay certain signals. Various design methods have been proposed and an excellent survey of FDF is presented in [6].

The frequency response error, i.e. the error of the frequency response deviated from the ideal one, is the conventional frequency-domain criterion to evaluate the performance of FDFs. Up to now most of the studies is focused on optimizing the frequency response errors. However, it is noteworthy that the functionality of fractional delayer or FDFs is to predict the values which lie somewhere between two samples. Thus the time-domain error, i.e. error of the actual delayed signal deviated from the ideal delayed signal, should also be a very important evaluation criterion and should be given more attention [7].

From Parseval's theorem, reducing the frequency response error of FDF will reduce the time-domain error. However, the FDFs based on Shannon sampling scheme will lead to an irreducible error when the normalized radian frequency approaches $\pi$ [8]. FDF utilizing Hermite interpolation with an analog differentiator is an effective improvement because it is based on multi-channel sampling scheme and nearly eliminates the frequency response error when the normalized radian frequency approaches $\pi$ [9].

Although FDF based on Hermite interpolation with an analog differentiator achieves high interpolation accuracy in time-domain, it requires more samplers and more analog differentiators than Shannon sampling scheme. The synchronization of all the samplers and the design of analog differentiator with high precision are intractable tasks when the sampling rate and the signal frequency are very high. Thus, a simplified scheme, which utilizes Hermite interpolation with a digital differentiator, has been proposed in [9]. This simplified scheme is based on Shannon sampling scheme. However, the time-domain error greatly rises when the signal frequency approaches the Nyquist rate. This phenomenon appears because the frequency response of digital differentiator is undesirable.

To realize a lower time-domain error while maintaining a simple sampling module, in this letter we propose a novel fractional delayer utilizing Hermite interpolation with Carathéodory representation. The Carathéodory representation asserts existence and uniqueness of the representation of a finite discrete signal in terms of exponential sums, and the samples of differential signal could be estimated from the samples of original signal only [10]. After the samples of the original signal and differential signal have been obtained, Hermite interpolation is implemented. Simulation results for complex single-frequency signal, complex multi-frequency signal, complex ultra-wideband (UWB) linear frequency modulated (LFM) and UWB nonlinear frequency modulated (UWB NLFM) signal demonstrate that the time-domain error of the proposed method is much lower than that of the existing Hermite interpolation 
method with digital differentiator. Meanwhile, our method is based on Shannon sampling scheme and requires only one sampler.

The rest of this paper is organized as follows. Section 2 introduces the scheme of FDF utilizing Hermite interpolation. Section 3 presents the proposed modified fractional delayer. To demonstrate the superiority of the proposed method in terms of time-domain error, computer simulations are conducted utilizing three types of signals, and the results are given in Sec. 4. Finally, Section 5 offers some conclusions drawn on the basis of simulation results.

\section{Fractional Delayer Utilizing Hermite Interpolation}

For FDFs, minimizing the frequency response error deviated from the ideal one will reduce the time-domain error. The ideal frequency response of FDF is given in Laakso's tutorial paper [6], and is given below

$$
H_{\mathrm{ID}}\left(\mathrm{e}^{\mathrm{j} \omega}\right)=\mathrm{e}^{-\mathrm{j} \omega D}
$$

where $D$ denotes delay, a positive real number in the desired range. In case of FIR filters, $D$ should be around $L$, where $2 L$ is the order of FIR filter. $\omega$ is the normalized radian frequency. The subscript ID means ideal.

However, there is an irreducible error at $\omega=\pi$ for conventional designs based on the Shannon sampling scheme. Thus, FDF utilizing Hermite interpolation, which is based on multi-channel sampling technique, is a representative improvement.

Figure 1 shows the scheme of fractional delayer utilizing Hermite interpolation with analog differentiator. $x[n]$ represent the samples of the original continuous-time signal $x(t)$, and $x^{\prime}[n]$ denote the samples of $x^{\prime}(t)$, where $x^{\prime}(t)$ is the first-order derivative of $x(t) . H_{1}(z)$ and $H_{2}(z)$ are two FIR digital filters. The coefficients of the two digital FIR filters are given in Tseng's paper [9, equations (18) and (20)], and are expressed in (2a) and (2b)

$$
\begin{gathered}
h_{1}[l]=[1-2(l-D) r(l)]\left(\prod_{i=0, i \neq l}^{2 L} \frac{D-i}{l-i}\right)^{2}, \\
h_{2}[l]=(l-D)\left(\prod_{i=0, i \neq l}^{2 L} \frac{D-i}{l-i}\right)^{2}
\end{gathered}
$$

where

$$
r[l]=\sum_{s=0, s \neq l}^{L} \frac{1}{s-l}
$$

and $l=0,1, \ldots, 2 L .2 L$ is the order Hermite fractional delay filter set. The output of this scheme, i.e. the actual delayed signal, denoted by $y[n]$, is

$$
y[n] \approx x[n-D]=x[n] * h_{1}[n]+x^{\prime}[n] * h_{2}[n]
$$

where $*$ denotes the convolution operation.

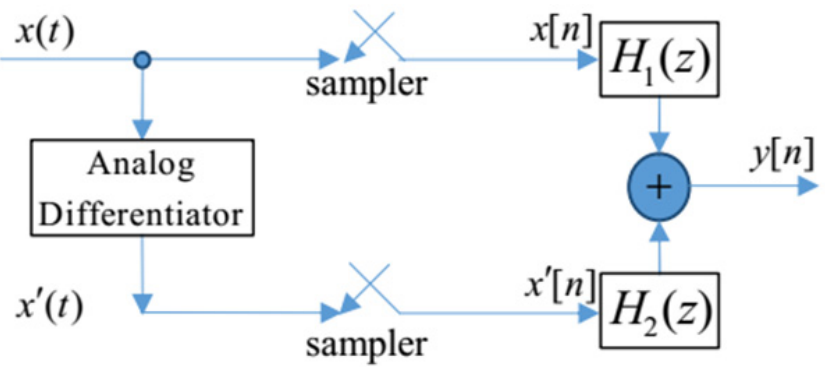

Fig. 1. The scheme of fractional delayer utilizing Hermite interpolation utilizing analog differentiator.

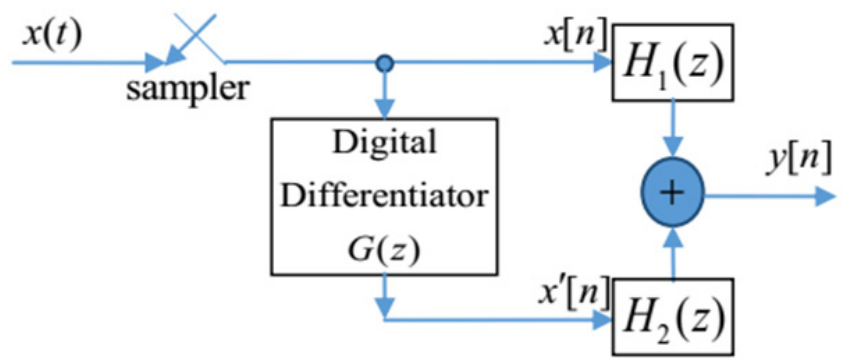

Fig. 2. The simplified scheme of fractional delayer utilizing Hermite interpolation with digital differentiator.

The frequency response of Hermite fractional delay filter with analog differentiator, which approximates the ideal frequency response of FDF, is given in (5)

$$
H\left(\mathrm{e}^{\mathrm{j} \omega}\right)=H_{1}\left(\mathrm{e}^{\mathrm{j} \omega}\right)+\mathrm{j} \omega H_{2}\left(\mathrm{e}^{\mathrm{j} \omega}\right)
$$

where $H_{1}\left(\mathrm{e}^{\mathrm{j} \omega}\right)=\sum_{l=0}^{2 L} h_{1}[l] \mathrm{e}^{-\mathrm{j} \omega l}$ and $H_{2}\left(\mathrm{e}^{\mathrm{j} \omega}\right)=\sum_{l=0}^{2 L} h_{2}[l] \mathrm{e}^{-\mathrm{j} \omega l}$, i.e. the frequency response of $h_{1}[l]$ and $h_{2}[l]$ respectively.

In Fig. 1 , the derivative samples $x^{\prime}[n]$ are obtained by using an analog differentiator combining a sampler. However, an analog differentiator is difficult to implement with high precision, and this sampling scheme is more complicated than Shannon sampling scheme. Thus, a simplified scheme, which utilizes Hermite interpolation with digital differentiator has been proposed in [9], and is shown in Fig. 2.

The simplified scheme has only one sampler. The digital differentiator $G(z)$ is designed to fit the ideal frequency response of the first order differentiator and the derivative signal $x^{\prime}[n]$ is obtained from the discrete signal $x[n]$ directly. The two FIR filters $H_{1}(z)$ and $H_{2}(z)$ are the same ones mentioned above. The output of this scheme, denoted by $y[n]$, is given below

$$
y[n] \approx x[n-D]=x[n] * h_{1}[n]+x[n] *\left\{h_{2}[n] * g[n]\right\}
$$

where $g[n]$ denote the coefficients of $G(z)$.

The frequency response of the simplified Hermite fractional delay filter is given below

$$
H\left(\mathrm{e}^{\mathrm{j} \omega}\right)=H_{1}\left(\mathrm{e}^{\mathrm{j} \omega}\right)+G\left(\mathrm{e}^{\mathrm{j} \omega}\right) H_{2}\left(\mathrm{e}^{\mathrm{j} \omega}\right)
$$

where $G\left(\mathrm{e}^{\mathrm{j} \omega}\right)$ is the frequency response of digital differentiator. 
For both the schemes, each interpolation point, $y[n]$, is created from $2 L+1$ samples of $x[n]$ and $2 L+1$ samples of $x^{\prime}[n]$.

\section{The New Fractional Delayer}

\subsection{On Approximation of Derivative Samples by Carathéodory Representation}

The Carathéodory representation theorem asserts existence and uniqueness of the representation of a finite sequence in terms of exponential sums [10], and the number of terms could be significantly reduced by allowing exponents and coefficients to be complex-valued [11]. follows:

We reformulate the Carathéodory representation as

Given one group containing $2 L+1$ uniformly-spaced samples of a smooth function $x(t)$ on the interval $\left[n / f_{\mathrm{s}},(n+2 L) / f_{\mathrm{s}}\right]$, and a target accuracy $\varepsilon>0$, we can find the minimal number $K$ of complex weights $\omega_{k}$ and complex nodes $\tau_{k}$ such that

$$
\left|x[l]-\sum_{k=1}^{K} \omega_{k} \mathrm{e}^{\tau_{k} l}\right| \leq \varepsilon \quad \text { for each } l=n, n+1, \cdots, n+2 L
$$

where $x[l]$ are the samples of $x(t)$, and $x[l]=\left.x(t)\right|_{t=l / f_{s}}$. $n=0,1, \ldots, N-1-2 L$, and $N$ is the number of $x[n] . K$ is a positive integer and is usually taken from 1 to $L+1 . f_{\mathrm{s}}$ is the sampling rate.

The algorithm to obtain the nodes and weights above is as follows

1. Build a $(L+1) \times(L+1)$ Hankel matrix $\mathbf{H}_{k, l}=x_{k+l}$ where $x_{m}=x[m][12$, p. 204].

2. Find a vector $\mathbf{u}_{K}=\left(u_{0} u_{1} \ldots u_{L}\right)^{\mathrm{T}} \quad$ satisfying $\mathbf{H} \mathbf{u}_{K}=\sigma_{K} \overline{\mathbf{u}}_{K}$. The "bar" notation denotes conjugate operation and $\sigma_{K}$ is close to the target accuracy $\varepsilon$. To obtain $\sigma_{K}$ and $\mathbf{u}_{K}$, we can apply singular value decomposition (SVD) to $\mathbf{H}$. Then we label the first $K+1$ singular values in decreasing order $\sigma_{0} \geq \sigma_{1} \geq \ldots \geq \sigma_{K}$, where $\sigma_{K} / \sigma_{0} \approx \varepsilon$, and the corresponding singular vector obtained is $\mathbf{u}_{K}$.

3. Compute the roots of the polynomial $u(z)=\sum_{l=0}^{L} u_{l} z^{-l}$ where $l=1,2, \ldots, L$. We denote these roots by $\gamma_{l}$ and select all the $K$ roots close to the unit circle, i.e. the $\gamma_{l}$ satisfying ||$\gamma_{l}|-1|<\kappa$ where $\kappa$ is a small constant much close to zero. Thus $\gamma_{l}=\exp \left(\tau_{l}\right)$.

4. Obtain the $K$ weights by solving the overdetermined Vandermonde system below

$$
x[l]=\sum_{k=1}^{K} \omega_{k} \mathrm{e}^{\tau_{k} l}, \quad \text { for each } l=n, n+1, \cdots, n+2 L .
$$

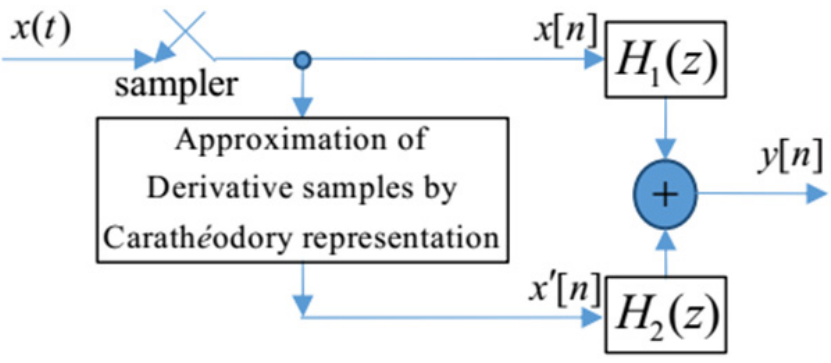

Fig. 3. The modified scheme of fractional delayer.

The algorithm of Carathéodory representation requires $O\left((2 L+1)(\log (2 L+1))^{2}\right)$ operations that can be seen in [10], [11].

With the complex weights $\omega_{k}$ and complex nodes $\tau_{k}$ in hand, the derivative samples of $x(t)$ on the interval $\left[n,(n+2 L) / f_{\mathrm{s}}\right]$, denoted by $x^{\prime}[l]$, can be approximated below

$$
x^{\prime}[l]=\sum_{k=1}^{K} \tau_{k} \omega_{k} \mathrm{e}^{\tau_{k} l} \quad \text { for each } l=n, n+1, \cdots, n+2 L .
$$

Thus, obtaining the derivative samples requires neither an analog differentiator nor a sampler.

\subsection{The Modified Scheme}

Based on the analysis above, we propose a modified fractional delayer. Figure 3 shows the modified scheme. $H_{1}(z)$ and $H_{2}(z)$ are two FIR filters mentioned in Sec. 2. $2 L+1$ is the length of both these filters. Having being reformulated each group containing $2 L+1$ samples of $x[n]$ by Carathéodory representation, the corresponding $2 L+1$ samples of $x^{\prime}[n]$ are approximated by (9). Then a fractional delayer based on Hermite interpolation could be implemented, and the output of this system is given in (4).

The Hermite interpolation requires $O(2 L+1)$ operations and the total computational complexity of the proposed method is $O(2 L+1)+O\left((2 L+1)(\log (2 L+1))^{2}\right)$.

\section{Simulations}

In this section, we devise four simulations to verify the validity and superiority of the proposed method. The first simulation addresses a complex single-frequency signal while the second simulation discusses a complex multifrequency signal. In the third case, we use a UWB LFM signal, while in the fourth case, we use a UWB nonlinear LFM (NLFM) signal. Minimizing the time-domain error is our objective. In each simulation, we compare the interpolation error for Hermite interpolation method with digital differentiator in [9], Hermite interpolation method with analog differentiator in [9] and the proposed method.

The time-domain error, or say interpolation error, can be expressed below

$$
e(n)=20 \log _{10}\left|y[n]-y_{\mathrm{IFD}}[n]\right|
$$


where $y[n]$ is the actual delayed signal and $y_{\mathrm{IFD}}[n]$ is the ideal delayed signal. $L=8$, so the order of Hermite interpolation filter set is 16 .

\subsection{Complex Single-Frequency Signal}

The discrete complex single-frequency signal $x[n]$ and the ideal delayed signal $y_{\mathrm{IFD}}[n]$ are given below

$$
\begin{gathered}
x[n]=\exp \left(\mathrm{j} 2 \pi f_{0} \frac{n}{f_{s}}\right), \\
y_{\mathrm{IFD}}[n]=\exp \left(\mathrm{j} 2 \pi f_{0} \frac{n-D}{f_{s}}\right)
\end{gathered}
$$

where $f_{0}=2.19 \mathrm{GHz}, n=0,1, \ldots, N-1$ and $N=1000$. The accuracy $\varepsilon$ is $10^{-3}$ and $K=2$. Figure 4 shows the time-domain errors for the three methods mentioned above when $D=8.1$. Table 1 shows the maximum interpolation error using the three methods mentioned for $D=$ $8.1,8.2, \ldots, 8.8,8.9$. The results indicate that the proposed method has achieved a much lower interpolation error than Hermite interpolation method with digital differentiator when the signal frequency approaches the Nyquist rate.

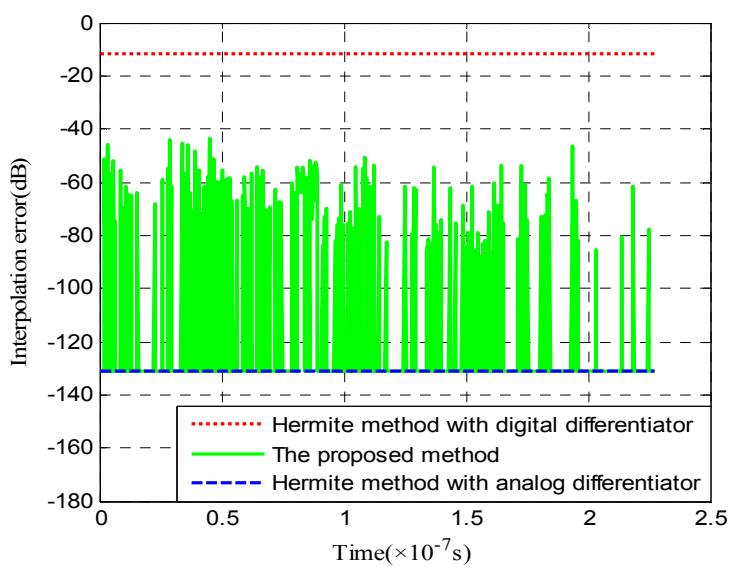

Fig. 4. The interpolation errors of the three methods tested by complex single-frequency signal when $D=8.1$. Dotted, solid, dashed lines represent the Hermite interpolation method with digital differentiator, the modified method and the Hermite interpolation method with analog differentiator, respectively.

\subsection{Complex Multi-Frequency Signal}

The discrete complex multi-frequency signal $x[n]$ and the ideal delayed signal $y_{\mathrm{IFD}}[n]$ are given below

$$
\begin{aligned}
x[n] & =\sum_{m=1}^{M} \exp \left(\mathrm{j} 2 \pi f_{m} \frac{n}{f_{s}}\right), \\
y_{\mathrm{IFD}}[n] & =\sum_{m=1}^{M} \exp \left(\mathrm{j} 2 \pi f_{m} \frac{n-D}{f_{s}}\right)
\end{aligned}
$$

where $M$ is the number of frequencies and $M=6 . f_{1}=$ $0.365 \mathrm{GHz}, f_{2}=0.73 \mathrm{GHz}, f_{3}=1.095 \mathrm{GHz}, f_{4}=1.46 \mathrm{GHz}$, $f_{5}=1.825 \mathrm{GHz}, f_{6}=2.19 \mathrm{GHz}, \quad n=0,1, \ldots, N-1$ and $N=1000$. The accuracy $\varepsilon$ is $10^{-3}$ and $K=7$. Figure 5 shows the interpolation error for three methods mentioned above when $D=8.1$. Table 2 shows the maximum interpolation errors using three methods mentioned when $D=$ $8.1,8.2, \ldots, 8.8,8.9$. The results show the performance of our method has much higher interpolation accuracy than the Hermite interpolation method with digital differentiator. The time-domain error tested by complex multi-frequency signal is also lower than one tested by complex single-frequency signal.

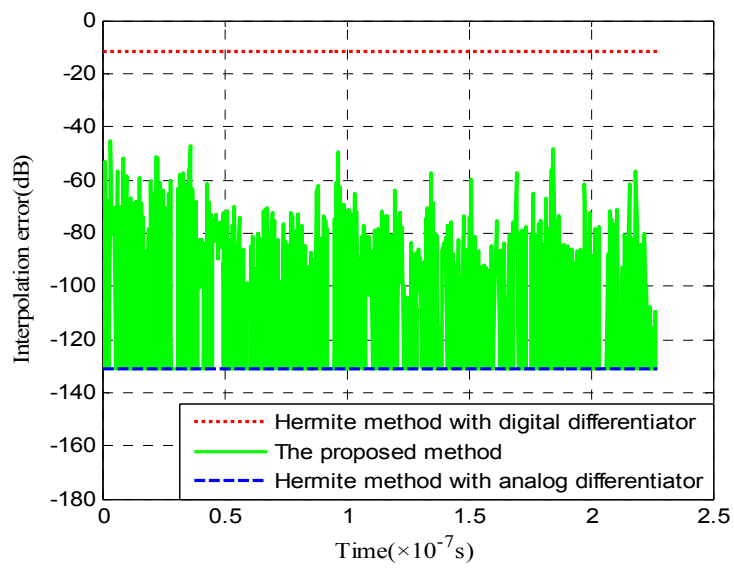

Fig. 5. The interpolation errors of the three methods tested by complex multi-frequency signal when $D=8.1$. Dotted solid, dashed lines represent the Hermite interpolation method with digital differentiator, the modified method and the Hermite interpolation method with analog differentiator, respectively.

\begin{tabular}{|c|c|c|c|}
\hline & \multicolumn{2}{|c|}{ Maximum interpolation error(dB) } \\
\hline$D$ & $\begin{array}{c}\text { Fractional delayer based on } \\
\text { Hermite interpolation method } \\
\text { with digital differentiator }\end{array}$ & $\begin{array}{c}\text { The proposed } \\
\text { fractional delayer }\end{array}$ & $\begin{array}{c}\text { Fractional delayer based on } \\
\text { Hermite interpolation method } \\
\text { with analog differentiator }\end{array}$ \\
\hline 8.1 & -11.7 & -43.6 & -132.4 \\
\hline 8.2 & -6.1 & -38.0 & -122.0 \\
\hline 8.3 & -3.3 & -35.2 & -115.5 \\
\hline 8.4 & -1.9 & -33.8 & -112.6 \\
\hline 8.5 & -1.5 & -33.4 & -111.5 \\
\hline 8.6 & -1.9 & -33.8 & -112.2 \\
\hline 8.7 & -3.3 & -35.2 & -114.7 \\
\hline 8.8 & -6.1 & -38.0 & -120.2 \\
\hline 8.9 & -11.6 & -43.7 & -130.8 \\
\hline
\end{tabular}

Tab. 1. Maximum interpolation errors for complex single-frequency signal utilizing the three methods. 


\subsection{Complex UWB LFM Signal}

The discrete complex UWB LFM signal $x[n]$ and the ideal delayed signal $y_{\mathrm{IFD}}[n]$ are given below

$$
\begin{gathered}
x(n)=\exp \left\{\mathrm{j} 2 \pi\left[f_{0} \frac{n}{f_{s}}+\mu\left(\frac{n}{f_{s}}\right)^{2}\right]\right\}, \\
y_{\mathrm{IFD}}(n)=\exp \left\{\mathrm{j} 2 \pi\left[f_{0} \frac{n-D}{f_{s}}+\mu\left(\frac{n-D}{f_{s}}\right)^{2}\right]\right\}
\end{gathered}
$$

where $\mu=B / t_{0} . B$ is the signal bandwidth and $B=0.5 \mathrm{GHz}$ $t_{0}$ is the duration of the signal and $t_{0}=N / f_{\mathrm{s}} . f_{0}=1.69 \mathrm{GHz}$. $n=0,1, \ldots, N-1$ and $N=1000$. The accuracy $\varepsilon$ is $10^{-3}$ and $K=8$. Figure 6 shows the interpolation errors utilizing three methods mentioned above when $D=8.1$. Table 3 shows the maximum interpolation errors using three methods mentioned for $D=8.1,8.2, \ldots, 8.8,8.9$. In contrast, the proposed delayer could effectively split the signal into its constituent frequencies and accurately sniff these frequencies. So, our method could estimate the derivative samples $x^{\prime}[n]$ much more accurately than using digital differentiator. Thus, our scheme is suitable to delay UWB LFM signals.

\subsection{Complex UWB NLFM Signal}

NLFM signals can solve the contradiction between the main lobe width and the side lobe level, so they have

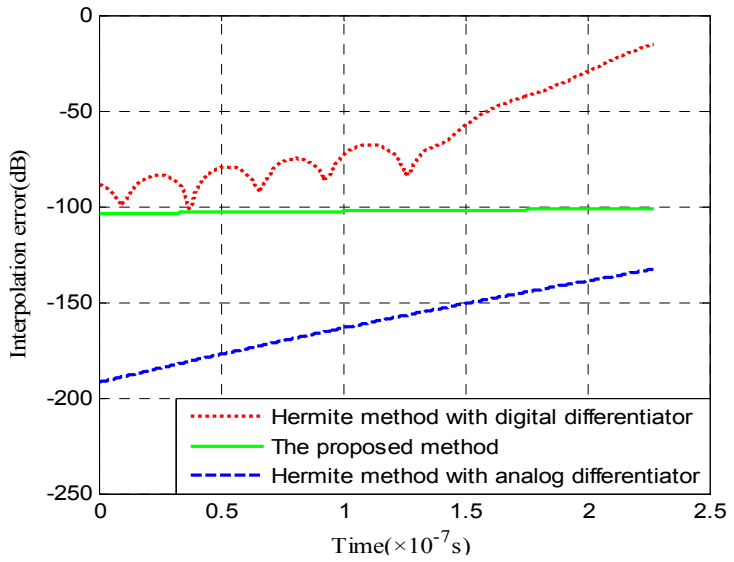

Fig. 6. The interpolation errors of the three methods tested by complex UWB LFM signal when $D=8.1$. Dotted, solid, dashed lines represent the Hermite interpolation method with digital differentiator, the modified method and the Hermite interpolation method with analog differentiator, respectively.

been widely concerned in the pulse compression radars and UWB radars. One of most the most common UWB NFLM signals is the tangent FM signal. The discrete complex UWB NLFM signal $x[n]$ and the ideal delayed signal $y_{\mathrm{IFD}}[n]$ are given below

$$
x[n]=\exp \left\{\mathrm{j} 2 \pi\left[f_{0} \frac{n}{f_{s}}+\frac{\beta t_{0}}{v \pi} \ln \left(\cos \left(\frac{v \pi}{t_{0}}\left(\frac{n}{f_{s}}-\frac{t_{0}}{2}\right)\right)\right)\right]\right\},
$$

\begin{tabular}{|c|c|c|c|}
\hline & \multicolumn{3}{|c|}{ Maximum interpolation error(dB) } \\
\hline$D$ & $\begin{array}{c}\text { Fractional delayer based on } \\
\text { Hermite interpolation method } \\
\text { with digital differentiator }\end{array}$ & $\begin{array}{c}\text { The proposed } \\
\text { fractional delayer }\end{array}$ & $\begin{array}{c}\text { Fractional delayer based on } \\
\text { Hermite interpolation method } \\
\text { with analog differentiator }\end{array}$ \\
\hline 8.1 & -11.8 & -72.7 & -132.4 \\
\hline 8.2 & -6.2 & -67.3 & -122.1 \\
\hline 8.3 & -3.4 & -64.5 & -115.5 \\
\hline 8.4 & -2 & -63.1 & -112.5 \\
\hline 8.5 & -1.6 & -62.7 & -111.5 \\
\hline 8.6 & -2 & -63.1 & -112.1 \\
\hline 8.7 & -3.4 & -64.6 & -114.7 \\
\hline 8.8 & -6.1 & -67.2 & -120.2 \\
\hline 8.9 & -11.7 & -72.9 & -130.9 \\
\hline
\end{tabular}

Tab. 2. Maximum interpolation errors for complex multi-frequency signal utilizing the three methods.

\begin{tabular}{|c|c|c|c|}
\hline & \multicolumn{3}{|c|}{ Maximum interpolation error(dB) } \\
\hline$D$ & $\begin{array}{c}\text { Fractional delayer based on } \\
\text { Hermite interpolation method } \\
\text { with digital differentiator }\end{array}$ & $\begin{array}{c}\text { The proposed } \\
\text { fractional delayer }\end{array}$ & $\begin{array}{c}\text { Fractional delayer based on } \\
\text { Hermite interpolation method } \\
\text { with analog differentiator }\end{array}$ \\
\hline 8.1 & -15.4 & -101.2 & -132.9 \\
\hline 8.2 & -9.8 & -92.9 & -121.7 \\
\hline 8.3 & -7.0 & -86.7 & -116.0 \\
\hline 8.4 & -5.6 & -83.3 & -113.1 \\
\hline 8.5 & -5.2 & -81.2 & -112.0 \\
\hline 8.6 & -5.6 & -80.2 & -112.8 \\
\hline 8.7 & -7.1 & -80.4 & -115.2 \\
\hline 8.8 & -9.8 & -82.1 & -120.5 \\
\hline 8.9 & -15.3 & -86.7 & -131.3 \\
\hline
\end{tabular}

Tab. 3. Maximum interpolation errors for complex UWB LFM signal utilizing the three methods. 


\begin{tabular}{|c|c|c|c|}
\hline & \multicolumn{3}{|c|}{ Maximum interpolation error(dB) } \\
\hline$D$ & $\begin{array}{c}\text { Fractional delayer based on } \\
\text { Hermite interpolation method } \\
\text { with digital differentiator }\end{array}$ & $\begin{array}{c}\text { The proposed } \\
\text { fractional delayer }\end{array}$ & $\begin{array}{c}\text { Fractional delayer based on } \\
\text { Hermite interpolation method } \\
\text { with analog differentiator }\end{array}$ \\
\hline 8.1 & -16.2 & -79.9 & -137.6 \\
\hline 8.2 & -10.6 & -75.0 & -126.3 \\
\hline 8.3 & -8.8 & -71.8 & -121.2 \\
\hline 8.4 & -7.4 & -70.0 & -118.2 \\
\hline 8.5 & -6.9 & -69.0 & -117.2 \\
\hline 8.6 & -7.4 & -68.9 & -117.8 \\
\hline 8.7 & -8.8 & -69.9 & -120.2 \\
\hline 8.8 & -11.5 & -72.2 & -125.5 \\
\hline 8.9 & -17.1 & -77.3 & -136.3 \\
\hline
\end{tabular}

Tab. 4. Maximum interpolation errors for complex UWB NLFM signal utilizing the three methods.

$$
\exp _{I F D}\left[\mathrm{j} 2 \pi\left[f_{0} \frac{n-D}{f_{s}}+\frac{\beta t_{0}}{v \pi} \ln \left(\cos \left(\frac{v \pi}{t_{0}}\left(\frac{n-D}{f_{s}}-\frac{t_{0}}{2}\right)\right)\right)\right]\right\} .
$$

$t_{0}$ is the duration of the signal and $t_{0}=N / f_{\mathrm{s}} . f_{0}=1.94 \mathrm{GHz}$. $n=0,1, \ldots, N-1$ and $N=1000 . \beta$ is modulation factor of the tangent FM signal above and $\beta$ is given as follows

$$
\beta=\frac{B}{2 \tan (v \pi / 2)}
$$

where $B$ is the signal bandwidth and $B=0.5 \mathrm{GHz} . v=0.5$. The accuracy $\varepsilon$ is $10^{-3}$ and $K=3$. Figure 7 shows the interpolation errors utilizing three methods mentioned above when $D=8.1$. Table 4 shows the maximum interpolation errors using three methods mentioned for $D=$ $8.1,8.2, \ldots, 8.8,8.9$. The results show that the performance of the proposed method has excellent interpolation accuracy and is suitable for UWB signals, especially in the high frequency region. When the signal frequency approximates $1.69 \mathrm{GHz}$, the frequency modulation slope of the NLFM signal is larger than that for the LFM signal, so the proposed scheme has a little higher interpolation error than using digital differentiator. Although the interpolation error is a bit higher, it is still less than $-70 \mathrm{~dB}$ and high interpolation accuracy is obtained.

\section{Conclusion}

In this letter, we proposed a novel fractional delayer utilizing Hermite interpolation with Carathéodory representation. The derivative samples are obtained by Carathéodory representation from the samples of the original signal, other than analog or digital differentiator. Thus, only one sampler is required. Theoretical analysis and simulation results for four types of signals demonstrate that our method has much lower interpolation error than Hermite interpolation method with digital differentiator. Moreover, the proposed method also has a much simpler sampling module than Hermite interpolation method with an analog differentiator.

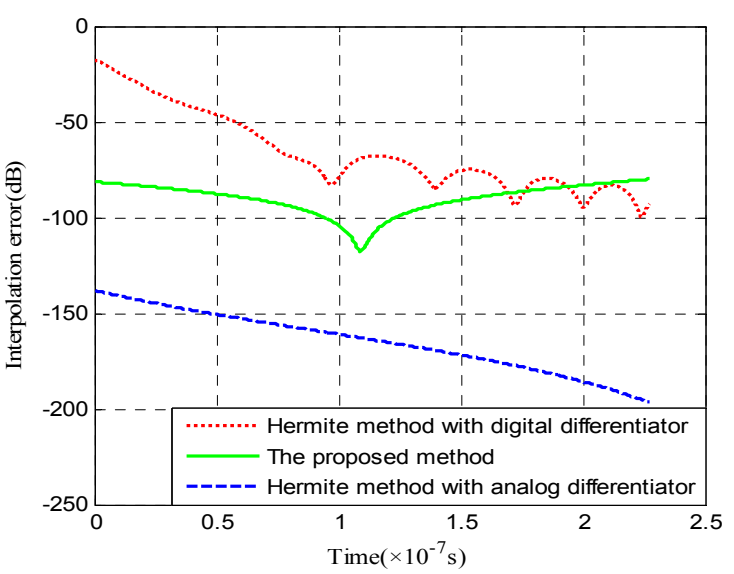

Fig. 7. The interpolation errors of the three methods tested by complex UWB NLFM signal when $D=8.1$. Dotted, solid, dashed lines represent the Hermite interpolation method with digital differentiator, the modified method and the Hermite interpolation method with analog differentiator, respectively.

\section{Acknowledgments}

This work is sponsored by the National Natural Science Foundation of China under grant No. 61271331 and 61571229.

\section{References}

[1] SIVANAND, S., YANG, J. F., KAVEH, M. Focusing filters for wide-band direction finding. IEEE Transactions on Signal Processing, 1991, vol. 39, no. 2, p. 437-445. DOI: $10.1109 / 78.80827$

[2] KROON, P., ATAL, B. S. On the use of pitch predictors with high temporal resolution. IEEE Transactions on Signal Processing, 1991, vol. 39, no. 3, p. 733-735. DOI: 10.1109/78.80859

[3] DENG, T.-B., SOONTORNWONG, P. Delay-error-constrained minimax design of all-pass variable-fractional-delay digital filters. Signal Processing, 2016, vol. 120, p. 438-447. DOI: 10.1016/j.sigpro.2015.10.002 
[4] DENG, T.-B., QIN, W. Improved bi-equiripple variable fractional delay filters. Signal Processing, 2014, vol. 94, no. 1, p. 300-307. DOI: $10.1016 /$ j.sigpro.2013.07.004

[5] SOONTORNWONG, P., CHIVAPREECHA, S. Pascalinterpolation-based non-integer delay filter and low-complexity realization. Radioengineering, 2015 , vol. 24 , no. 4, p. 1002-1012. DOI: $10.13164 /$ re.2015.1002

[6] LAAKSO, T. I., VALIMAKI, V., KARJALAINEN, M., et al. Splitting the unit delay-tools for fractional delay filter design. IEEE Signal Processing Magazine, 1996, vol. 13, no. 1, p. 30-60. DOI: $10.1109 / 79.482137$

[7] YU, Y.-J., XU W.-J. Investigation on the optimization criteria for the design of variable fractional delay filters. IEEE Transactions on Circuits and System II: Express Briefs, 2013, vol. 60, no. 8, p. 522-526. DOI: $10.1109 /$ TCSII.2013.2268239

[8] TSENG, C.-C., LEE S.-L. Design of wideband fractional delay filters using derivative sampling method. IEEE Transactions on Circuits and System I: Regular Papers, 2010, vol. 57, no. 8, p. 2087-2098. DOI: 10.1109/TCSI.2009.2037451

[9] TSENG, C.-C., LEE S.-L. Design of fractional delay filter using Hermite interpolation method. IEEE Transactions on Circuits and System I: Regular Papers, 2012, vol. 59, no. 7, p. 1458-1471. DOI: 10.1109/TCSI.2011.2177136

[10] BEYLKIN, G., MONZÓN, L. On generalized Gaussian quadratures for exponentials and their applications. Applied and Computational Harmonic Analysis, 2002, vol. 23, no. 3, p. 332-373. DOI: 10.1006/acha.2002.0380

[11] BEYLKIN, G., MONZÓN, L. On approximation of functions by exponential sums. Applied and Computational Harmonic Analysis. 2005, vol. 19, no. 1, p. 17-48. DOI: 10.1016/j.acha.2005.01.003

[12] HORN, R. A., JOHNSON, C. R. Matrix Analysis. Rev. New York (USA): Cambridge University Press, 1985. ISBN: 0521305861

\section{About the Authors ...}

Qiang DU was born in 1985. He received his M.Sc. from Nanjing University of Posts and Telecommunications in 2007. Currently he is working towards the Ph.D. degree at the School of Electronic and Optical Engineering, Nanjing University of Science and Technology, Nanjing, China. His research interests include array signal processing and ultra wideband (UWB) signal processing.

Yaoliang SONG (corresponding author) was born in 1960 He received his M.Sc. from the B.Eng., the M.Eng. and the D.Eng. degrees in Electrical Engineering from Nanjing University of Science and Technology, China, in 1983, 1986, and 2000, respectively. He had been a Researcher Fellow at the Dept. of Engineering Science at the University of Oxford from Sept. 2004 to Sept. 2005. Presently, he is a Professor at Nanjing University of Science and Technology, and is heading the UWB Radar Imaging Group. His major research interests are in UWB communications, UWB radar imaging, and advanced signal processing.

Zeeshan AHMAD was born in Pakistan in 1988. He received the B.E. degree in Electrical (Telecom) Engineering from the Bahria University, Islamabad, Pakistan, in 2011 and the M.E. degree in Electronics and Communication Engineering from Chongqing University, Chongqing, China, in 2014. Currently, he is enrolled in Nanjing University of Science and Technology, Nanjing, Jiangsu Province, China as a Ph.D. student. His research interests include array signal processing, ultra wideband, wideband and narrowband beamforming, radars, adaptive arrays, GPS and satellite navigation systems. 